\title{
Projected Gradient Methods for Nonnegative Matrix Factorization
}

\author{
Chih-Jen Lin
}

cjlin@csie.ntu.edu.tw

Department of Computer Science, National Taiwan University, Taipei 106, Taiwan

Nonnegative matrix factorization (NMF) can be formulated as a minimization problem with bound constraints. Although bound-constrained optimization has been studied extensively in both theory and practice, so far no study has formally applied its techniques to NMF. In this letter, we propose two projected gradient methods for NMF, both of which exhibit strong optimization properties. We discuss efficient implementations and demonstrate that one of the proposed methods converges faster than the popular multiplicative update approach. A simple Matlab code is also provided.

\section{Introduction}

Nonnegative matrix factorization (NMF) (Paatero \& Tapper 1994; Lee \& Seung, 1999) is useful for finding representations of nonnegative data. Given an $n \times m$ data matrix $V$ with $V_{i j} \geq 0$ and a prespecified positive integer $r<$ $\min (n, m)$, NMF finds two nonnegative matrices $W \in R^{n \times r}$ and $H \in R^{r \times m}$ such that

$$
V \approx W H
$$

If each column of $V$ represents an object, NMF approximates it by a linear combination of $r$ "basis" columns in $W$. NMF has been applied to many areas, such as finding basis vectors of images (Lee \& Seung, 1999), document clustering (Xu, Liu, \& Gong, 2003), and molecular pattern discovery (Brunet, Tamayo, Golub, \& Mesirov, 2004). Donoho and Stodden (2004) have addressed the theoretical issues associated with the NMF approach.

The conventional approach to find $W$ and $H$ is by minimizing the difference between $V$ and $W H$ :

$$
\begin{array}{rl}
\min _{W, H} & f(W, H) \equiv \frac{1}{2} \sum_{i=1}^{n} \sum_{j=1}^{m}\left(V_{i j}-(W H)_{i j}\right)^{2} \\
\text { subject to } & W_{i a} \geq 0, H_{b j} \geq 0, \quad \forall i, a, b, j .
\end{array}
$$


The inequalities such that variables are upper- and lower-bounded are referred to as bound constraints. Hence, equation 1.1 is a standard boundconstrained optimization problem. We also note that

$$
\sum_{i=1}^{n} \sum_{j=1}^{m}\left(V_{i j}-(W H)_{i j}\right)^{2}=\|V-W H\|_{F}^{2},
$$

where $\|\cdot\|_{F}$ is the Frobenius norm.

The most popular approach to solve equation 1.1 is the multiplicative update algorithm proposed by Lee and Seung (2001). It is simple to implement and often yields good results. At each iteration of this method, the elements of $W$ and $H$ are multiplied by certain factors. As the zero elements are not updated, all the components of $W$ and $H$ are strictly positive for all iterations. This type of strategy is contrary to the traditional boundconstrained optimization methods, which usually allow iterations to have bounded elements (i.e., zero elements in this case). Thus far, no study has formally applied bound-constrained optimization techniques to NMF. This letter investigates such methods in detail. Some earlier NMF studies require all $W^{\prime}$ 's column sums to be ones: $\sum_{i=1}^{n} W_{i a}=1, \forall a=1, \ldots, r$. The function value does not change because $f\left(W D, D^{-1} H\right)=f(W, H)$ for any $r \times r$ positive diagonal matrix $D$. With the inclusion of such additional constraints, equation 1.1 no longer remains a bounded problem. As adding these constraints may complicate the optimization procedures, we do not consider this modification in this study.

Among the existing bound-constrained optimization techniques, the projected gradient method is simple and effective. Although several researchers have used this method for NMF (Hoyer, 2002; Chu, Diele, Plemmons, \& Ragni, 2005; Shepherd, 2004), there is neither a systematic study nor an easy implementation comparable to that of the multiplicative update method. This letter presents a comprehensive study on using projected gradient methods for NMF. Several useful modifications lead to efficient implementations. While the multiplicative update method still lacks convergence results, our proposed methods exhibit strong optimization properties. We experimentally show that one of the proposed methods converges faster than the multiplicative update method. This new method is thus an attractive approach to solve NMF. We also provide a complete Matlab implementation.

Another popular NMF optimization formula is to minimize the (generalized) Kullback-Leibler divergence between $V$ and $W H$ (Lee \& Seung, 1999):

$$
\min _{W, H} \sum_{i=1}^{n} \sum_{j=1}^{m}\left(V_{i j} \log \frac{V_{i j}}{(W H)_{i j}}-V_{i j}+(W H)_{i j}\right)
$$

subject to $W_{i a} \geq 0, H_{b j} \geq 0, \forall i, a, b, j$. 
Strictly speaking, this formula is not a bound-constrained problem, which requires the objective function to be well defined at any point of the bounded region. The $\log$ function is not well defined if $V_{i j}=0$ or $(W H)_{i j}=0$. Hence, we do not consider this formulation in this study.

This letter is organized as follows. Section 2 discusses existing approaches for solving NMF problem 1.1 and presents several new properties. Section 3 introduces the projected gradient methods for bound-constrained optimization. Section 4 investigates specific but essential modifications for applying the proposed projected gradients methods to NMF. The stopping conditions in an NMF code are discussed in section 5. Experiments on synthetic and real data sets are presented in section 6 . The discussion and conclusions are presented in section 7. Appendix B contains the Matlab code of one of the proposed approaches. All source codes used in this letter are available online at http:/ / www.csie.ntu.edu.tw/ $\sim$ cjlin/nmf.

\section{Existing Methods and New Properties}

There are many existing methods for NMF. Some discussions are in Paatero (1999), but bound constraints are not rigorously handled. A more recent and complete survey is by Chu et al. (2005). This section briefly discusses some existing methods and presents several previously unmentioned observations.

To begin, we need certain properties of the NMF problem, equation 1.1. The gradient of the function $f(W, H)$ consists of two parts:

$$
\nabla_{W} f(W, H)=(W H-V) H^{T} \quad \text { and } \quad \nabla_{H} f(W, H)=W^{T}(W H-V),
$$

which are, respectively, partial derivatives to elements in $W$ and $H$. From the Karush-Kuhn-Tucker (KKT) optimality condition (e.g., Bertsekas, 1999), $(W, H)$ is a stationary point of equation 1.1 if and only if

$$
\begin{aligned}
& W_{i a} \geq 0, H_{b j} \geq 0 \\
& \nabla_{W} f(W, H)_{i a} \geq 0, \nabla_{H} f(W, H)_{b j} \geq 0 \\
& W_{i a} \cdot \nabla_{W} f(W, H)_{i a}=0, \text { and } H_{b j} \cdot \nabla_{H} f(W, H)_{b j}=0, \forall i, a, b, j .
\end{aligned}
$$

Optimization methods for NMF produce a sequence $\left\{W^{k}, H^{k}\right\}_{k=1}^{\infty}$ of iterations. Problem 1.1 is nonconvex and may have several local minima. A common misunderstanding is that limit points of the sequence are local minima. In fact, most nonconvex optimization methods guarantee only the stationarity of the limit points. Such a property is still useful, as any local minimum must be a stationary point. 
2.1 Multiplicative Update Methods. The most commonly used approach to minimize equation 1.1 is a simple multiplicative update method proposed by Lee and Seung (2001):

Algorithm 1: Multiplicative Update

1. Initialize $W_{i a}^{1}>0, H_{b j}^{1}>0, \forall i, a, b, j$.

2. For $k=1,2, \ldots$

$$
\begin{aligned}
& W_{i a}^{k+1}=W_{i a}^{k} \frac{\left(V\left(H^{k}\right)^{T}\right)_{i a}}{\left(W^{k} H^{k}\left(H^{k}\right)^{T}\right)_{i a}}, \quad \forall i, a \\
& H_{b j}^{k+1}=H_{b j}^{k} \frac{\left(\left(W^{k+1}\right)^{T} V\right)_{b j}}{\left(\left(W^{k+1}\right)^{T} W^{k+1} H^{k}\right)_{b j}}, \quad \forall b, j .
\end{aligned}
$$

This algorithm is a fixed-point type method: if $\left(W^{k} H^{k}\left(H^{k}\right)^{T}\right)_{i a} \neq 0$ and $W_{i a}^{k+1}=W_{i a}^{k}>0$, then

$$
\left(V\left(H^{k}\right)^{T}\right)_{i a}=\left(W^{k} H^{k}\left(H^{k}\right)^{T}\right)_{i a} \quad \text { implies } \quad \nabla_{W} f\left(W^{k}, H^{k}\right)_{i a}=0
$$

which is part of the KKT condition, equation 2.2. Lee and Seung (2001) have shown that the function value is nonincreasing after every update:

$$
f\left(W^{k+1}, H^{k}\right) \leq f\left(W^{k}, H^{k}\right) \quad \text { and } \quad f\left(W^{k+1}, H^{k+1}\right) \leq f\left(W^{k+1}, H^{k}\right)
$$

They claim that the limit of the sequence $\left\{W^{k}, H^{k}\right\}_{k=1}^{\infty}$ is a stationary point (i.e., a point satisfying the KKT condition, equation 2.2). However, Gonzales and Zhang (2005) indicate that this claim is wrong, as having equation 2.5 may not imply the convergence. Therefore, this multiplicative update method still lacks optimization properties.

To have algorithm 1 well defined, one must ensure that denominators in equations 2.3 and 2.4 are strictly positive. Moreover, if $W_{i a}^{k}=0$ at the $k$ th iteration, then $W_{i a}=0$ at all subsequent iterations. Thus, one should keep $W_{i a}^{k}>0$ and $H_{b j}^{k}>0, \forall k$. The following theorem discusses when this property holds:

Theorem 1. If $V$ has neither zero column nor row, and $W_{i a}^{1}>0$ and $H_{b j}^{1}>$ $0, \forall i, a, b, j$, then

$$
W_{i a}^{k}>0 \text { and } H_{b j}^{k}>0, \forall i, a, b, j, \forall k \geq 1
$$

The proof is straightforward, and is in appendix A.

If $V$ has zero columns or rows, a division by zero may occur. Even if theorem 1 holds, denominators close to zero may still cause numerical problems. Some studies, such as Piper, Pauca, Plemmons, and Giffin (2004) 
have proposed adding a small, positive number in the denominators of equations 2.3 and 2.4. We observe numerical difficulties in a few situations and provide more discussion in section 6.3.

Regarding the computational complexity, $V\left(H^{k}\right)^{T}$ and $\left(W^{k+1}\right)^{T} V$ in equation 2.3 and 2.4 are both $\mathrm{O}(\mathrm{nmr})$ operations. One can calculate the denominator in equation 2.3 by either

$$
(W H) H^{T} \quad \text { or } \quad W\left(H H^{T}\right) \text {. }
$$

The former takes $O(n m r)$ operations, but the latter costs $O\left(\left(\max (m, n) r^{2}\right)\right.$. As $r<\min (m, n)$, the latter is better. Similarly for equation 2.4, $\left(W^{T} W\right) H$ should be used. This discussion indicates the importance of having fewer $O(n m r)$ operations (i.e., $W H, W^{T} V$, or $V H^{T}$ ) in any NMF code.

In summary, the overall cost of algorithm 1 is

\#iterations $\times \mathrm{O}(\mathrm{nmr})$.

All time complexity analysis in this letter assumes that $V, W$, and $H$ are implemented as dense matrices.

2.2 Alternating Nonnegative Least Squares. From the nonincreasing property 2.6, algorithm 1 is a special case of a general framework, which alternatively fixes one matrix and improves the other: find $W^{k+1}$ such that $f\left(W^{k+1}, H^{k}\right) \leq f\left(W^{k}, H^{k}\right)$, and find $H^{k+1}$ such that $f\left(W^{k+1}, H^{k+1}\right) \leq$ $f\left(W^{k+1}, H^{k}\right)$. The extreme situation is to obtain the best point (Paatero, 1999; Chu et al., 2005):

Algorithm 2: Alternating Nonnegative Least Squares

1. Initialize $W_{i a}^{1} \geq 0, H_{b j}^{1} \geq 0, \forall i, a, b, j$.

2. For $k=1,2, \ldots$

$$
\begin{aligned}
& W^{k+1}=\arg \min _{W \geq 0} f\left(W, H^{k}\right) \\
& H^{k+1}=\arg \min _{H \geq 0} f\left(W^{k+1}, H\right) .
\end{aligned}
$$

This approach is the block coordinate descent method in bound-constrained optimization (Bertsekas, 1999), where sequentially one block of variables is minimized under corresponding constraints and the remaining blocks are fixed. For NMF, we have the simplest case of only two block variables $W$ and $H$. 
We refer to equations 2.8 or 2.9 as a subproblem in algorithm 2 . When one block of variables is fixed, a subproblem is indeed the collection of several nonnegative least-squares problems. From equation 2.9,

$$
H^{k+1} \text { 's } j \text { th column }=\min _{\mathbf{h} \geq \mathbf{0}}\left\|\mathbf{v}-W^{k+1} \mathbf{h}\right\|^{2},
$$

where $\mathbf{v}$ is the $j$ th column of $V$ and $\mathbf{h}$ is a vector variable. Chu et al. (2005) suggest projected Newton's methods (Lawson \& Hanson, 1974) to solve each problem (see equation 2.10). Clearly, solving subproblems 2.8 and 2.9 per iteration could be more expensive than the simple update in algorithm 1. Then algorithm 2 may be slower even though we expect that it better decreases the function value at each iteration. Efficient methods to solve subproblems are thus essential. Section 4.1 proposes using project gradient methods and discusses why they are suitable for solving subproblems in algorithm 2 .

Regarding the convergence of algorithm 2, one may think that it is a trivial result. For example, Paatero (1999) states that for the alternating nonnegative least-squares approach, no matter how many blocks of variables we have, the convergence is guaranteed. However, this issue deserves some attention. Past convergence analysis for block coordinate descent methods requires subproblems to have unique solutions (Powell, 1973; Bertsekas, 1999), but this property does not hold here: subproblems 2.8 and 2.9 are convex, but they are not strictly convex. Hence, these subproblems may have multiple optimal solutions. For example, when $H^{k}$ is the zero matrix, any $W$ is optimal for equation 2.8. Fortunately, for the case of two blocks, Grippo and Sciandrone (2000) have shown that this uniqueness condition is not needed. Directly from corollary 2 of Grippo and Sciandrone (2000), we have the following convergence result:

Theorem 2. Any limit point of the sequence $\left\{W^{k}, H^{k}\right\}$ generated by algorithm 2 is a stationary point of equation 1.1.

The remaining issue is whether the sequence $\left\{W^{k}, H^{k}\right\}$ has at least one limit point (i.e., there is at least one convergent subsequence). In optimization analysis, this property often comes from the boundedness of the feasible region, but our region under constraints $W_{i a} \geq 0$ and $H_{b j} \geq 0$ is unbounded. One can easily add a large upper bound to all variables in equation 1.1. As the modification still leads to a bound-constrained problem, algorithm 2 can be applied and theorem 2 holds. In contrast, it is unclear how to easily modify the multiplicative update rules if there are upper bounds in equation 1.1.

In summary, contrary to algorithm 1, which still lacks convergence results, algorithm 2 has nice optimization properties. 
2.3 Gradient Approaches. In Chu et al. (2005, sec. 3.3), several gradienttype approaches have been mentioned. In this section, we briefly discuss methods that select the step size along the negative gradient direction. By defining

$$
W_{i a}=E_{i a}^{2} \quad \text { and } \quad H_{b j}=F_{b j}^{2},
$$

Chu et al. (2005) reformulate equation 1.1 as an unconstrained optimization problem of variables $E_{i a}$ and $F_{b j}$. Then standard gradient descent methods can be applied. The same authors also mention that Shepherd (2004) uses

$$
\begin{aligned}
& W^{k+1}=\max \left(0, W^{k}-\alpha_{k} \nabla_{W} f\left(W^{k}, H^{k}\right)\right), \\
& H^{k+1}=\max \left(0, H^{k}-\alpha_{k} \nabla_{H} f\left(W^{k}, H^{k}\right)\right),
\end{aligned}
$$

where $\alpha_{k}$ is the step size. This approach is already a projected gradient method. However, in the above references, details are not discussed.

\section{Projected Gradient Methods for Bound-Constrained Optimization}

We consider the following standard form of bound-constrained optimization problems:

$$
\begin{array}{rl}
\min _{\mathbf{x} \in R^{n}} & f(\mathbf{x}) \\
\text { subject to } & l_{i} \leq x_{i} \leq u_{i}, \quad i=1, \ldots, n,
\end{array}
$$

where $f(\mathbf{x}): R^{n} \rightarrow R$ is a continuously differentiable function, and $\mathbf{1}$ and $\mathbf{u}$ are lower and upper bounds, respectively. Assume $k$ is the index of iterations. Projected gradient methods update the current solution $\mathbf{x}^{k}$ to $\mathbf{x}^{k+1}$ by the following rule:

$$
\mathbf{x}^{k+1}=P\left[\mathbf{x}^{k}-\alpha^{k} \nabla f\left(\mathbf{x}^{k}\right)\right]
$$

where

$$
P\left[x_{i}\right]= \begin{cases}x_{i} & \text { if } l_{i}<x_{i}<u_{i} \\ u_{i} & \text { if } x_{i} \geq u_{i} \\ l_{i} & \text { if } x_{i} \leq l_{i}\end{cases}
$$

maps a point back to the bounded feasible region. Variants of projected gradient methods differ on selecting the step size $\alpha^{k}$. We consider a simple and effective one called "Armijo rule along the projection arc" in Bertsekas (1999), which originates from Bertsekas (1976). The procedure is illustrated in algorithm 3 : 
Algorithm 3: A Projected Gradient Method for Bound-Constrained Optimization

1. Given $0<\beta<1,0<\sigma<1$. Initialize any feasible $\mathbf{x}^{1}$.

2. For $k=1,2, \ldots$

$$
\mathbf{x}^{k+1}=P\left[\mathbf{x}^{k}-\alpha_{k} \nabla f\left(\mathbf{x}^{k}\right)\right]
$$

where $\alpha_{k}=\beta^{t_{k}}$, and $t_{k}$ is the first nonnegative integer $t$ for which

$$
f\left(\mathbf{x}^{k+1}\right)-f\left(\mathbf{x}^{k}\right) \leq \sigma \nabla f\left(\mathbf{x}^{k}\right)^{T}\left(\mathbf{x}^{k+1}-\mathbf{x}^{k}\right)
$$

Condition 3.2, used in most proofs of projected gradient methods, ensures the sufficient decrease of the function value per iteration. By trying the step sizes $1, \beta, \beta^{2}, \ldots$, Bertsekas (1976) has proved that $\alpha_{k}>0$ satisfying equation 3.2 always exists and every limit point of $\left\{\mathbf{x}^{k}\right\}_{k=1}^{\infty}$ is a stationary point of equation 3.1. A common choice of $\sigma$ is 0.01 , and we consider $\beta=1 / 10$ in this letter. In the experiment section, 6.2 , we have some discussions about the choice of $\beta$.

Searching $\alpha_{k}$ is the most time-consuming operation in algorithm 3, so one should check as few step sizes as possible. Since $\alpha_{k-1}$ and $\alpha_{k}$ may be similar, a trick in Lin and Moré (1999) uses $\alpha_{k-1}$ as the initial guess and then either increases or decreases it in order to find the largest $\beta^{t_{k}}$ satisfying equation 3.2. Moreover, with nonnegative $t_{k}$, algorithm 4 may be too conservative by restricting $\alpha_{k} \leq 1$. Sometimes a larger step more effectively projects variables to bounds at one iteration. Algorithm 4 implements a better initial guess of $\alpha$ at each iteration and allows $\alpha$ to be larger than one:

Algorithm 4: An Improved Projected Gradient Method

1. Given $0<\beta<1,0<\sigma<1$. Initialize any feasible $\mathbf{x}^{1}$. Set $\alpha_{0}=1$.

2 For $k=1,2, \ldots$

(a) Assign $\alpha_{k} \leftarrow \alpha_{k-1}$

(b) If $\alpha_{k}$ satisfies equation 3.2, repeatedly increase it by

$\alpha_{k} \leftarrow \alpha_{k} / \beta$

until either $\alpha_{k}$ does not satisfy equation 3.2 or $\mathbf{x}\left(\alpha_{k} / \beta\right)=\mathbf{x}\left(\alpha_{k}\right)$.

Else repeatedly decrease $\alpha_{k}$ by

$\alpha_{k} \leftarrow \alpha_{k} \cdot \beta$

until $\alpha_{k}$ satisfies equation 3.2.

(c) Set

$$
\mathbf{x}^{k+1}=P\left[\mathbf{x}^{k}-\alpha_{k} \nabla f\left(\mathbf{x}^{k}\right)\right]
$$


The convergence result has been proved in, for example, Calamai and Moré (1987). One may think that finding $\alpha$ with the largest function reduction leads to faster convergence:

$$
\alpha_{k} \equiv \arg \min _{\alpha \geq 0} f\left(P\left[\mathbf{x}^{k}-\alpha \nabla f\left(\mathbf{x}^{k}\right)\right]\right)
$$

The convergence of selecting such an $\alpha_{k}$ is proved in McCormick and Tapia (1972). However, equation 3.3 is a piecewise function of $\alpha$, which is difficult to be minimized.

A major obstacle for minimizing bounded problems is to identify free (i.e., $l_{i}<x_{i}<u_{i}$ ) and active (i.e., $x_{i}=l_{i}$ or $u_{i}$ ) components at the convergent stationary point. Projected gradient methods are considered effective for doing so since they are able to add several active variables at a single iteration. However, once these sets have been (almost) identified, the problem in a sense reduces to an unconstrained one, and the slow convergence of gradient-type methods may occur. We will explain in section 4.1 that for NMF problems, this issue may not be serious.

\section{Projected Gradient Methods for NMF}

We apply projected gradient methods to NMF in two situations. The first case solves nonnegative least-squares problems discussed in section 2.2. The second case directly minimizes equation 1.1. Both approaches have convergence properties following from theorem 2 and Calamai and Moré (1987), respectively. Several modifications specific to NMF will be presented.

\subsection{Alternating Nonnegative Least Squares Using Projected Gradient} Methods. Section 2.2 indicates that algorithm 2 relies on efficiently solving subproblems 2.8 and 2.9, each of which is a bound-constrained problem. We propose using project gradient methods to solve them.

Subproblem 2.9 consists of $m$ independent nonnegative least-square problems (see equation 2.10), so one could solve them separately, a situation suitable for parallel environments. However, in a serial setting, treating them together is better for the following reasons:

- These nonnegative least-square problems are closely related as they share the same constant matrices $V$ and $W^{k+1}$ in equation 2.10.

- Working on the whole $H$ but not its individual columns implies that all operations are matrix based. Since finely tuned numerical linear algebra codes have better speed-up on matrix than on vector operations, we can thus save computational time. 
For a simpler description of our method, we focus on equation 2.9 and rewrite it as

$$
\begin{aligned}
\min _{H} & \bar{f}(H) \equiv \frac{1}{2}\|V-W H\|_{F}^{2} \\
\text { subject to } & H_{b j} \geq 0, \quad \forall b, j .
\end{aligned}
$$

Both $V$ and $W$ are constant matrices in equation 4.1. If we concatenate $H^{\prime}$ s columns to a vector vec $(H)$, then

$$
\begin{aligned}
f(H) & =\frac{1}{2}\|V-W H\|_{F}^{2} \\
& =\frac{1}{2} \operatorname{vec}(H)^{T}\left[\begin{array}{lll}
W^{T} W & & \\
& \ddots & \\
& & W^{T} W
\end{array}\right] \operatorname{vec}(H)+H^{\prime} \text { s linear terms. }
\end{aligned}
$$

The Hessian matrix (i.e., second derivative) of $\bar{f}(H)$ is block diagonal, and each block $W^{T} W$ is an $r \times r$ positive semidefinite matrix. As $W \in R^{n \times r}$ and $r \ll n, W^{T} W$ and the whole Hessian matrix tend to be well conditioned, a good property for optimization algorithms. Thus, gradient-based methods may converge fast enough. A further investigation of this conjecture is in the experiment section, 6.2 .

The high cost of solving the two subproblems, equations 2.8 and 2.9, at each iteration is a concern. It is thus essential to analyze the time complexity and find efficient implementations. Each subproblem requires an iterative procedure, whose iterations are referred to as subiterations. When using algorithm 4 to solve equation 4.1 , we must maintain the gradient

$$
\nabla \bar{f}(H)=W^{T}(W H-V)
$$

at each subiteration. Following the discussion near equation 2.7, one should calculate it by $\left(W^{T} W\right) H-W^{T} V$. Constant matrices $W^{T} W$ and $W^{T} V$ can be computed respectively in $O\left(n r^{2}\right)$ and $O(n m r)$ operations before running subiterations.

The main computational task per subiteration is to find a step size $\alpha$ such that the sufficient decrease condition 3.2 is satisfied. Assume $\bar{H}$ is the current solution. To check if

$$
\tilde{H} \equiv P[\bar{H}-\alpha \nabla \bar{f}(\bar{H})]
$$

satisfies equation 3.2, calculating $\bar{f}(\tilde{H})$ takes $O(n m r)$ operations. If there are $t$ trials of $\tilde{H}^{\prime}$ s, the computational cost $O(t n m r)$ is prohibitive. We propose 
the following strategy to reduce the cost: for a quadratic function $f(\mathbf{x})$ and any vector $\mathbf{d}$,

$$
f(\mathbf{x}+\mathbf{d})=f(\mathbf{x})+\nabla f(\mathbf{x})^{T} \mathbf{d}+\frac{1}{2} \mathbf{d}^{T} \nabla^{2} f(\mathbf{x}) \mathbf{d}
$$

Hence, for two consecutive iterations $\overline{\mathbf{x}}$ and $\tilde{\mathbf{x}}$, equation 3.2 can be written as

$$
(1-\sigma) \nabla f(\overline{\mathbf{x}})^{T}(\tilde{\mathbf{x}}-\overline{\mathbf{x}})+\frac{1}{2}(\tilde{\mathbf{x}}-\overline{\mathbf{x}})^{T} \nabla^{2} f(\overline{\mathbf{x}})(\tilde{\mathbf{x}}-\overline{\mathbf{x}}) \leq 0
$$

Now $f(H)$ defined in equation 4.1 is quadratic, so equation 3.2 becomes

$$
(1-\sigma)\langle\nabla \bar{f}(\bar{H}), \tilde{H}-\bar{H}\rangle+\frac{1}{2}\left\langle\tilde{H}-\bar{H},\left(W^{T} W\right)(\tilde{H}-\bar{H})\right\rangle \leq 0,
$$

where $\langle\cdot, \cdot\rangle$ is the sum of the component-wise product of two matrices. The major operation in equation 4.3 is the matrix product $\left(W^{T} W\right) \cdot(\tilde{H}-\bar{H})$, which takes $O\left(m r^{2}\right)$. Thus, the cost $O(t n m r)$ of checking equation 3.2 is significantly reduced to $O\left(t m r^{2}\right)$. With the cost $O(n m r)$ for calculating $W^{T} V$ in the beginning, the complexity of using algorithm 4 to solve subproblem 4.1 is

$$
O(n m r)+\# \text { sub-iterations } \times O\left(t m r^{2}\right),
$$

where $t$ is the average number of checking equation 3.2 at each subiteration.

The pseudocode for optimizing equation 4.1 is in section B.2. We can use the same procedure to obtain $W^{k+1}$ by rewriting equation 2.9 as a form similar to equation 4.1 :

$$
\bar{f}(W) \equiv \frac{1}{2}\left\|V^{T}-H^{T} W^{T}\right\|_{F}^{2}
$$

where $V^{T}$ and $H^{T}$ are constant matrices.

The overall cost to solve equation 1.1 is

$$
\text { \#iterations } \times\left(O(n m r)+\# \text { sub-iterations } \times O\left(t m r^{2}+t n r^{2}\right)\right) \text {. }
$$

At each iteration, there are two $O(n m r)$ operations: $V\left(H^{k}\right)^{T}$ and $\left(W^{k+1}\right)^{T} V$, the same as those in the multiplicative update method. If $t$ and \#subiterations are small, this method is efficient.

To reduce the number of subiterations, a simple but useful technique is to warm-start the solution procedure of each subproblem. At final iterations, 
$\left(W^{k}, H^{k}\right)^{\prime}$ s are all similar, so $W^{k}$ is an effective initial point for solving equation 2.8 .

4.2 Directly Applying Projected Gradients to NMF. We may directly apply algorithm 4 to minimize equation 1.1 . Similar to solving the nonnegative least-squares problems in section 4.1 , the most expensive operation is checking the sufficient decrease condition, equation 3.2. From the current solution $(\bar{W}, \bar{H})$, we simultaneously update both matrices to $(\tilde{W}, \tilde{H})$ :

$$
(\tilde{W}, \tilde{H}) \equiv P\left[(\bar{W}, \bar{H})-\alpha\left(\nabla_{W} f(\bar{W}, \bar{H}), \nabla_{H} f(\bar{W}, \bar{H})\right)\right]
$$

As $f(W, H)$ is not a quadratic function, equation 4.2 does not hold. Hence, the trick, equation 4.3 , cannot be applied to save the computational time. Then, calculating $f(\tilde{W}, \tilde{H})=\frac{1}{2}\|V-\tilde{W} \tilde{H}\|_{F}^{2}$ takes $O(n m r)$ operations. The total computational cost is

\section{\#iterations $\times O($ tnmr $)$,}

where $t$ is the average number of condition 3.2 checked per iteration.

Given any random initial $\left(W^{1}, H^{1}\right)$, if $\left\|V-W^{1} H^{1}\right\|_{F}^{2}>\|V\|_{F}^{2}$, very often after the first iteration, $\left(W^{2}, H^{2}\right)=(0,0)$ causes the algorithm to stop. The solution $(0,0)$ is a useless stationary point of equation 1.1. A simple remedy is to find a new initial point $\left(W^{1}, \bar{H}^{1}\right)$ such that $f\left(W^{1}, \bar{H}^{1}\right)<f(0,0)$. By solving $\bar{H}^{1}=\arg \min _{H \geq 0} f\left(W^{1}, H\right)$ using the procedure described in section 4.1, we have

$$
\left\|V-W^{1} \bar{H}^{1}\right\|_{F}^{2} \leq\left\|V-W^{1} \cdot 0\right\|_{F}^{2}=\|V\|_{F}^{2} .
$$

The strict inequality generally holds, so $f\left(W^{1}, \bar{H}^{1}\right)<f(0,0)$.

\section{Stopping Conditions}

In all algorithms mentioned so far, we did not specify when the procedure should stop. Several implementations of the multiplicative update method (e.g., Hoyer, 2004) have an infinite loop, which must be interrupted by users after a time or iteration limit. Some researchers (e.g., Brunet, 2004) check the difference between recent iterations. If the difference is small enough, the procedure stops. However, such a stopping condition does not reveal whether a solution is close to a stationary point. In addition to a time or iteration limit, standard conditions to check the stationarity should also be included in NMF software. Moreover, in alternating least squares, each subproblem involves an optimization procedure, which needs a stopping condition as well. 
In bound-constrained optimization, a common condition to check if a point $\mathbf{x}^{k}$ is close to a stationary point is the following (Lin \& Moré, 1999):

$$
\left\|\nabla^{P} f\left(\mathbf{x}^{k}\right)\right\| \leq \epsilon\left\|\nabla f\left(\mathbf{x}^{1}\right)\right\|,
$$

where $\nabla^{P} f\left(\mathbf{x}^{k}\right)$ is the projected gradient defined as

$$
\nabla^{P} f(\mathbf{x})_{i} \equiv \begin{cases}\nabla f(\mathbf{x})_{i} & \text { if } l_{i}<x_{i}<u_{i} \\ \min \left(0, \nabla f(\mathbf{x})_{i}\right) & \text { if } x_{i}=l_{i}, \\ \max \left(0, \nabla f(\mathbf{x})_{i}\right) & \text { if } x_{i}=u_{i} .\end{cases}
$$

This condition follows from an equivalent form of the KKT condition for bounded problems: $l_{i} \leq x_{i} \leq u_{i}, \forall i$, and

$$
\left\|\nabla^{P} f(\mathbf{x})\right\|=\mathbf{0} \text {. }
$$

For NMF, equation 5.1 becomes

$$
\left\|\nabla^{P} f\left(W^{k}, H^{k}\right)\right\|_{F} \leq \epsilon\left\|\nabla f\left(W^{1}, H^{1}\right)\right\|_{F} .
$$

For alternating least squares, each subproblem 2.8 or 2.9 requires a stopping condition as well. Ideally, the condition for them should be related to the global one for equation 1.1, but a suitable condition is not obvious. For example, we cannot use the same stopping tolerance in equation 5.3 for subproblems. A user may specify $\epsilon=0$ and terminate the code after a certain time or iteration limit. Then the same $\epsilon=0$ in solving the first subproblem will cause algorithm 2 to keep running at the first iteration. We thus use the following stopping conditions for subproblems. The returned matrices $W^{k+1}$ and $H^{k+1}$ from the iterative procedures of solving the subproblems 2.8 and 2.9 should, respectively, satisfy

$$
\left\|\nabla_{W}^{P} f\left(W^{k+1}, H^{k}\right)\right\|_{F} \leq \bar{\epsilon}_{W}, \quad \text { and } \quad\left\|\nabla_{H}^{P} f\left(W^{k+1}, H^{k+1}\right)\right\|_{F} \leq \bar{\epsilon}_{H},
$$

where we set

$$
\bar{\epsilon}_{W}=\bar{\epsilon}_{H} \equiv \max \left(10^{-3}, \epsilon\right)\left\|\nabla f\left(W^{1}, H^{1}\right)\right\|_{F}
$$

in the beginning and $\epsilon$ is the tolerance in equation 6.3. If the projected gradient method for solving equation 2.8 stops without any iterations, we decrease the stopping tolerance by

$$
\bar{\epsilon}_{W} \leftarrow \bar{\epsilon}_{W} / 10
$$

For subproblem $2.9, \bar{\epsilon}_{H}$ is reduced in a similar way. 


\section{Experiments}

We compare four methods discussed in this letter and refer to them in the following way:

- mult: The multiplicative update method described in section 2.1

- alspgrad: Alternating nonnegative least squares using the projected gradient method for each subproblem (see section 4.1)

- pgrad: A direct use of the projected gradient method on equation 1.1 (see section 4.2)

- Isqnonneg: Using Matlab command Isqnonneg to solve $m$ problems (see equation 2.10) in the alternating least-squares framework

All implementations are in Matlab (http://www.mathworks.com). We conduct experiments on an Intel Xeon $2.8 \mathrm{GHz}$ computer. Results of using synthetic and real data are presented in the following subsections. All source codes for experiments are available online at http:/ /www.csie.ntu. edu.tw/ cjlin/nmf.

6.1 Synthetic Data. We consider three problem sizes: $(m, r, n)=$ $(25,5,25),(50,10,250)$, and $(100,20,500)$. The matrix $V$ is randomly generated by the normal distribution (mean 0 and standard deviation 1 )

$$
V_{i j}=|N(0,1)|
$$

The initial $\left(W^{1}, H^{1}\right)$ is constructed in the same way, and all four methods share the same initial point. These methods may converge to different points due to the nonconvexity of the NMF problem, equation 1.1. To have a fair comparison, for the same $V$ we try 30 different initial $\left(W^{1}, H^{1}\right)$ and report the average results. As synthetic data may not resemble practical problems, we leave detailed analysis of the proposed algorithms in section 6.2, which considers real data.

We set $\epsilon$ in equation 6.3 to be $10^{-3}, 10^{-4}, 10^{-5}$, and $10^{-6}$ in order to investigate the convergence speed to a stationary point. We also impose a time limit of 1000 seconds and a maximal number of 8000 iterations on each method. As Isqnonneg takes long computing time, we run it only on the smallest data. Due to the slow convergence of mult and pgrad, for $\epsilon=10^{-6}$ we run only alspgrad.

Results of average time, number of iterations, and objective values are in Table 1. For small problems, Table 1 shows that all four methods give similar objective values as $\epsilon \rightarrow 0$. The method Isqnonneg is rather slow, a result supporting our argument in section 4.1 that a matrix-based approach is better than a vector-based one. For larger problems, when $\epsilon=10^{-5}$, mult and pgrad often exceed the maximal number of iterations. Clearly, mult quickly 


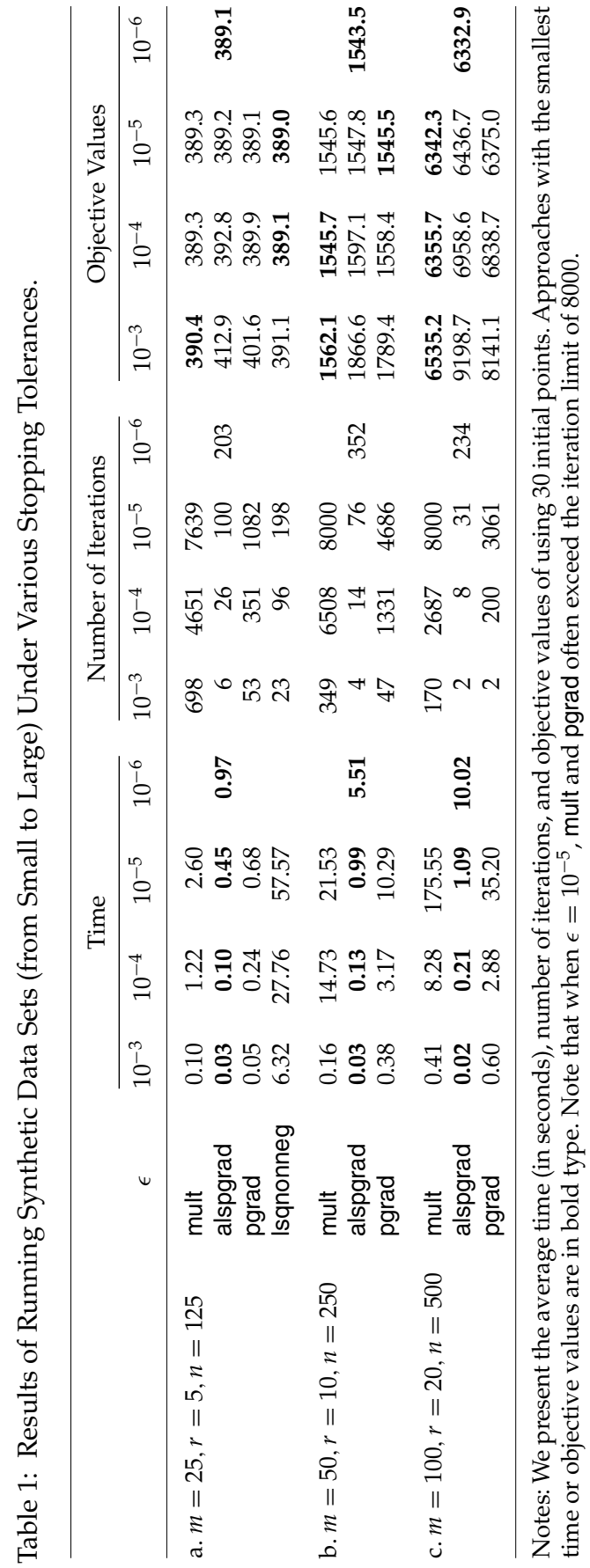


Table 2: Image Data: Average Objective Values and Projected-Gradient Norms of Using 30 Initial Points Under Specified Time Limits.

\begin{tabular}{|c|c|c|c|c|c|c|c|c|c|}
\hline \multirow{3}{*}{\multicolumn{2}{|c|}{$\begin{array}{l}\text { Problem } \\
\text { Size }(n r m) \\
\text { Time limit (in seconds) }\end{array}$}} & \multicolumn{2}{|c|}{ CBCL } & \multicolumn{3}{|c|}{ ORL } & \multicolumn{3}{|c|}{ Natural } \\
\hline & & $361 \quad 49$ & 2429 & 10,304 & 25 & 400 & 288 & 72 & 10,000 \\
\hline & & 25 & 50 & 25 & & 50 & & 25 & 50 \\
\hline \multirow[t]{2}{*}{ Objective value } & mult & 963.81 & 914.09 & 16.14 & & 14.31 & 370,797 . & 7.31 & $353,709.28$ \\
\hline & alspgrad & 923.77 & 870.18 & 14.34 & & 13.82 & 377,167 . & 7.27 & $352,355.64$ \\
\hline \multirow[t]{2}{*}{$\left\|\nabla f^{P}(W, H)\right\|_{F}$} & mult & 488.72 & 327.28 & 19.37 & & 9.30 & 54,534 . & 4.09 & $21,985.99$ \\
\hline & alspgrad & 230.67 & 142.13 & 4.82 & & 4.33 & 19,357 . & 7.03 & 4974.84 \\
\hline
\end{tabular}

Note: Smaller values are in bold type.

decreases the objective value in the beginning but slows down in the end. In contrast, alspgrad has the fastest final convergence. For the two larger problems, it gives the smallest objective value under $\epsilon=10^{-6}$ but takes less time than that by mult under $\epsilon=10^{-5}$. Due to the poor performance of pgrad and Isqnonneg, subsequently we focus on comparing mult and alspgrad.

6.2 Image Data. We consider three image problems used in Hoyer (2004):

- The CBCL (MIT Center for Biological and Computional Learning) face image database (http://cbcl.mit.edu/cbcl/software-datasets/ FaceData2.html).

- ORL (Olivetti Research Laboratory) face image database (http:// www.cl.cam.ac.uk/research/dtg/attarchive/facedatabase.html).

- Natural image data set (Hoyer, 2002).

All settings are the same as those in Hoyer (2004). We compare objective values and projected-gradient norms of mult and alspgrad after running 25 and 50 seconds. Table 2 presents average results of using 30 random initial points. For all three problems, alspgrad gives smaller objective values. While mult may quickly lower the objective value in the beginning, alspgrad catches up very soon and has faster final convergence. Results here are consistent with the findings in section 6.1. Regarding the projected-gradient norms, those by alspgrad are much smaller. Hence, solutions by alspgrad are closer to stationary points.

To further illustrate the slow final convergence of mult, Figure 1 checks the relation between the running time and the objective value. The CBCL set with the first of the 30 initial $\left(W^{1}, H^{1}\right)$ is used. The figure clearly demonstrates that mult very slowly decreases the objective value at final iterations.

The number of subiterations for solving equations 2.8 and 2.9 in alspgrad is an important issue. First, it is related to the time complexity analysis. Second, section 4.1 conjectures that the number should be small as $W^{T} W$ 


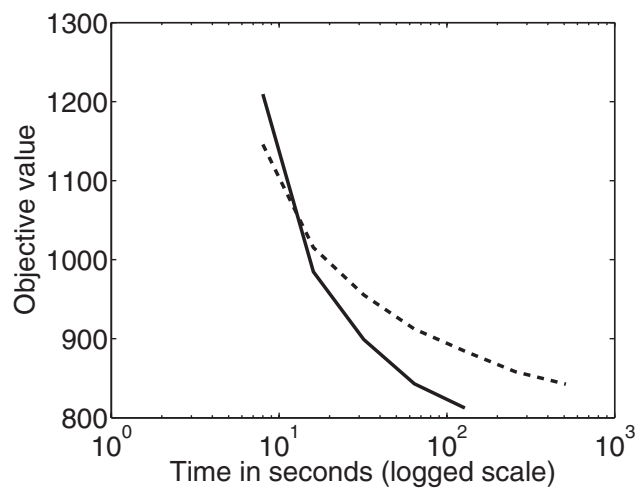

Figure 1: Time (seconds in log scale) versus objective values for mult (dashed line) and alspgrad (solid line).

Table 3: Number of Subiterations and Condition Numbers in Solving Equations 2.8 and 2.9 of alspgrad.

\begin{tabular}{|c|c|c|c|c|c|c|}
\hline \multirow{2}{*}{$\begin{array}{l}\text { Problem } \\
\text { Time limit (in seconds) }\end{array}$} & \multicolumn{2}{|c|}{ CBCL } & \multicolumn{2}{|c|}{ ORL } & \multicolumn{2}{|c|}{ Natural } \\
\hline & 25 & 50 & 25 & 50 & 25 & 50 \\
\hline$W$ : \# subiterations & 34.51 & 47.81 & 9.93 & 11.27 & 21.94 & 27.54 \\
\hline $\operatorname{cond}\left(H H^{T}\right)$ & 224.88 & 231.33 & 76.44 & 71.75 & 93.88 & 103.64 \\
\hline$H:$ \# subiterations & 11.93 & 18.15 & 6.84 & 7.70 & 3.13 & 4.39 \\
\hline $\operatorname{cond}\left(W^{T} W\right)$ & 150.89 & 124.27 & 478.35 & 129.00 & 38.49 & 17.19 \\
\hline
\end{tabular}

Notes: For subiterations, we calculate (total subiterations)/(total iterations) under each initial point and report the average of 30 values. For condition numbers, we find the median at all iterations, and report the average. Note that $H H^{T}\left(W^{T} W\right)$ corresponds to the Hessian of minimizing $W(H)$.

and $H H^{T}$ are generally well conditioned. Table 3 presents the number of subiterations and the condition numbers of $W^{T} W$ and $H H^{T}$. Compared to gradient-based methods in other scenarios, the number of subiterations is relatively small. Another projected gradient method pgrad discussed in Table 1 easily takes hundreds or thousands of iterations. For condition numbers, both the CBCL and natural sets have $r<n<m$, so $H H^{T}$ tends to be better conditioned than $W^{T} W$. ORL has the opposite as $r<m<n$. All condition numbers are small, and this result confirms our earlier conjecture. For ORL, $\operatorname{cond}\left(W^{T} W\right)>\operatorname{cond}\left(H H^{T}\right)$, but the number of subiterations on solving $W$ is more. One possible reason is the different stopping tolerances for solving equations 2.8 and 2.9 .

In the implementation of alspgrad, there is a parameter $\beta$, which is the rate of reducing the step size to satisfy the sufficient decrease condition, equation 3.2. It must be between 0 and 1 , and for the above experiments, 
Table 4: Text Data: Average Objective Values and Projected-Gradient Norms of Using 30 Initial Points Under Specified Time Limits.

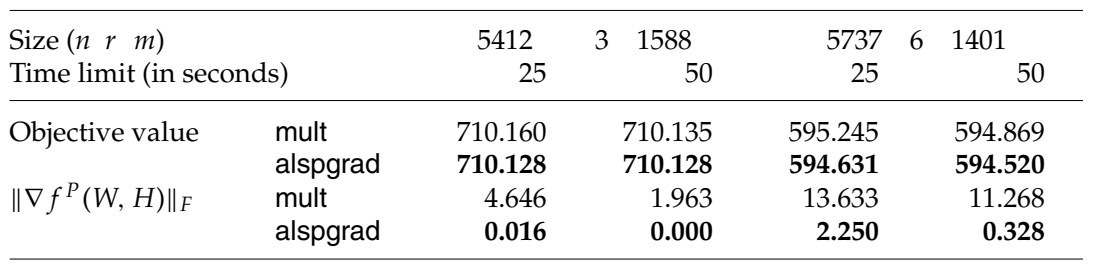

Notes: Smaller values are in bold type. Due to the unbalanced class distribution, interestingly the random selection of six classes results in fewer documents (i.e., $m$ ) than that of selecting three classes.

we use $\beta=0.1$. One may wonder about the effect of using other $\beta$. Clearly, a smaller $\beta$ more aggressively reduces the step size, but it may also cause a step size that in the end is too small. We consider the CBCL set with the first of the 30 initial $\left(W^{1}, H^{1}\right)$ (i.e., the setting to generate Figure 1, and check the effect of using $\beta=0.5$ and 0.1 . In both cases, alspgrad works well, but the one using 0.1 slightly more quickly reduces the function value. Therefore, $\beta=0.5$ causes too many checks for the sufficient decrease condition, equation 3.2. The cost per iteration is thus higher.

6.3 Text Data. NMF is useful for document clustering, so we next consider a text set RCV1 (Lewis, Yang, Rose, \& Li, 2004). This set is an archive of manually categorized newswire stories from Reuters Ltd. The collection has been fully preprocessed, including removing stop words, stemming, and transforming into vector space models. Each vector, cosine normalized, contains features of logged TF-IDF (term frequency, inverse document frequency). Training and testing splits have been defined. We remove documents in the training set that are associated with more than one class and obtain a set of 15,933 instances in 101 classes. We further remove classes with fewer than five documents. Using $r=3$ and 6 , we then randomly select $r$ classes of documents to construct the $n \times m$ matrix $V$, where $n$ is the number of the vocabulary set and $m$ is the number of documents. Some words never appear in the selected documents and cause zero rows in $V$. We remove them before experiments. The parameter $r$ is the number of clusters that we intend to assign documents to. Results of running mult and alspgrad by 25 and 50 seconds are in Table 4 . Again, alspgrad gives smaller objective values. In addition, projected-gradient norms of alspgrad are smaller.

In section 2.1, we mentioned that mult is well defined if theorem 1 holds. Now $V$ is a sparse matrix with many zero elements since words appearing in a document are only a small subset of the whole vocabulary set. Thus, some columns of $V$ are close to zero vectors, and for a few situations, numerical 
difficulties occur. In contrast, we do not face such problems for projected gradient methods.

\section{Discussion and Conclusions}

We discuss some future issues and draw conclusions.

7.1 Future Issues. As resulting $W$ and $H$ usually have many zero components, NMF is said to produce a sparse representation of the data. To achieve better sparseness, some studies, such as Hoyer (2002) and Piper et al. (2004) add penalty terms to the NMF objective function:

$$
\frac{1}{2} \sum_{i=1}^{n} \sum_{j=1}^{m}\left(V_{i j}-(W H)_{i j}\right)^{2}+\alpha\|W\|_{F}^{2}+\beta\|H\|_{F}^{2},
$$

where $\alpha$ and $\beta$ are positive numbers. Besides the Frobenius norm, which is quadratic, we can also use a linear penalty function,

$$
\alpha \sum_{i, a} W_{i a}+\beta \sum_{b, j} H_{b j}
$$

Our proposed methods can be used for such formulations. As penalty parameters $\alpha$ and $\beta$ only indirectly control the sparseness, Hoyer (2004) proposes a scheme to directly specify the desired sparsity. It is interesting to investigate how to incorporate projected gradient methods in such frameworks.

7.2 Conclusions. This letter proposes two projected gradient methods for NMF. The one solving least-squares subproblems in algorithm 2 leads to faster convergence than the popular multiplicative update method. Its success is due to our following findings:

- Subproblems in algorithm 2 for NMF generally have well-conditioned Hessian matrices (i.e., second derivative) due to the property $r \ll$ $\min (n, m)$. Hence, projected gradients converge quickly, although they use only the first-order information.

- The cost of selecting step sizes in the projected gradient method for subproblem 4.1 is significantly reduced by some reformulations that again use the property $r \ll \min (n, m)$.

Therefore, taking special NMF properties is crucial when applying an optimization method to NMF. 
Roughly speaking, optimization methods are between the following two extreme situations:

$$
\begin{aligned}
& \text { Low cost per iteration; } \\
& \text { slow convergence. }
\end{aligned} \begin{aligned}
& \text { High cost per iteration; } \\
& \text { fast convergence. }
\end{aligned}
$$

For example, Newton's methods are expensive per iteration but have very fast final convergence. Approaches with low cost per iteration usually decrease the objective value more quickly in the beginning, a nice property enjoyed by the multiplicative update method for NMF. Based on our analysis, we feel that the multiplicative update is very close to the first extreme. The proposed method of alternating least squares using projected gradients tends to be more in between. With faster convergence and strong optimization properties, it is an attractive approach for NMF.

\section{Appendix A: Proof of Theorem 1}

When $k=1$, equation 2.6 holds by the assumption of this theorem. Using induction, if equation 2.6 is correct at $k$, then at $(k+1)$, clearly denominators of equations 2.3 and 2.4 are strictly positive. Moreover, as $V$ has neither zero column nor row, both numerators are strictly positive as well. Thus, equation 2.6 holds at $(k+1)$, and the proof is complete.

\section{Appendix B: Matlab Code}

\section{B.1 Main Code for alspgrad (Alternating Nonnegative Least Squares Using Projected Gradients)}

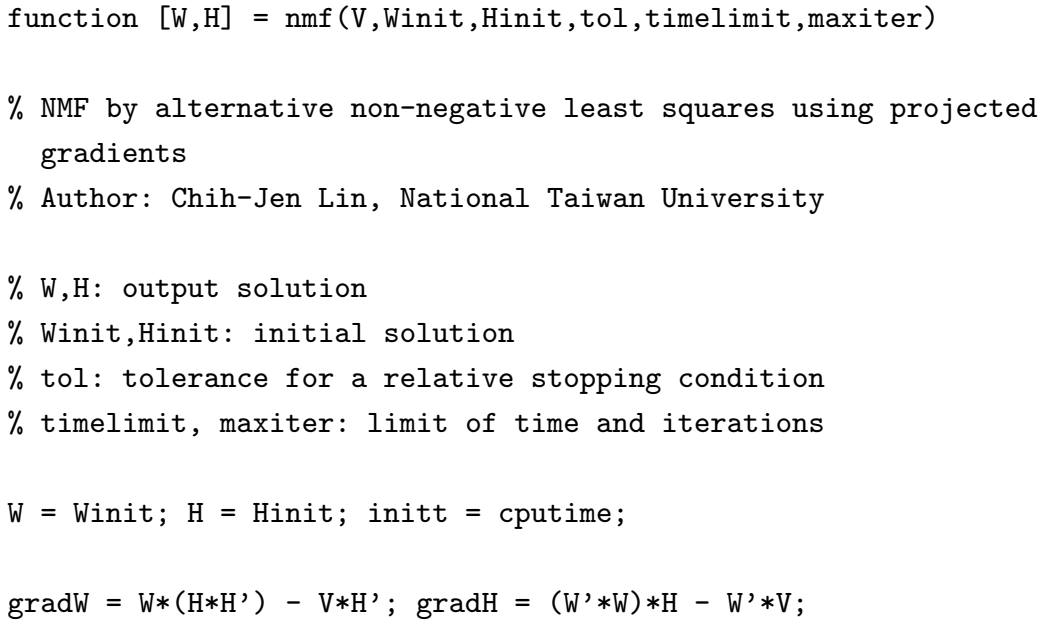




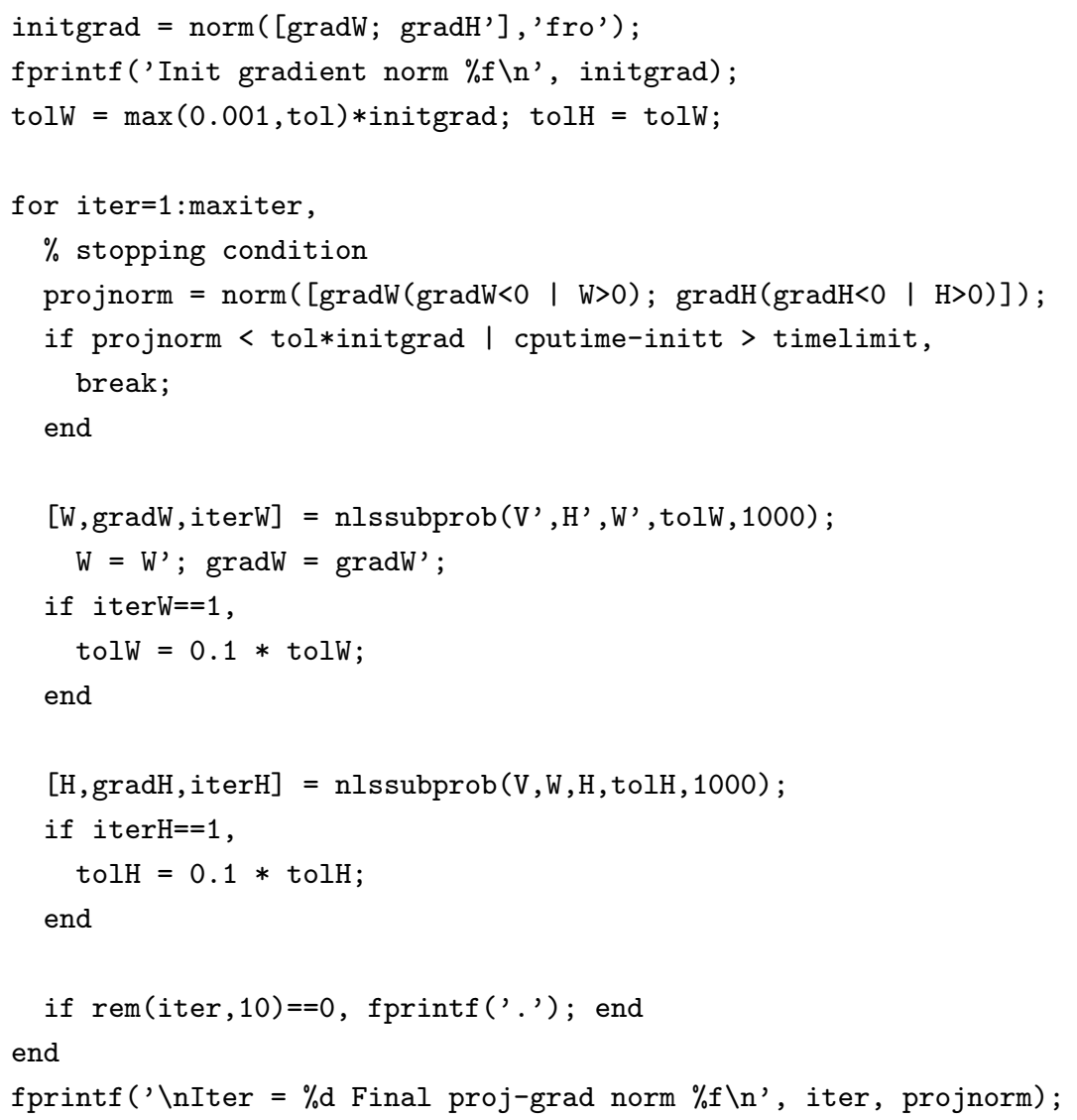

B.2 Solving Subproblem 4.1 by the Projected Gradient Algorithm 4.

function $[\mathrm{H}, \operatorname{grad}$, iter $]=\mathrm{nl} \operatorname{ssubprob}(\mathrm{V}, \mathrm{W}$, Hinit, tol, maxiter $)$

$\% \mathrm{H}$, grad: output solution and gradient

$\%$ iter: \#iterations used

$\% \mathrm{~V}, \mathrm{~W}$ : constant matrices

$\%$ Hinit: initial solution

$\%$ tol: stopping tolerance

$\%$ maxiter: limit of iterations

$\mathrm{H}=$ Hinit; $\mathrm{WtV}=\mathrm{W}^{\prime} * \mathrm{~V} ; \mathrm{WtW}=\mathrm{W}^{\prime} * \mathrm{~W} ;$

alpha $=1 ;$ beta $=0.1$; 




\section{Acknowledgments}

I thank Marco Sciandrone for pointing out the convergence of two-block coordinate descent methods in Grippo \& Sciandrone (2000) and helpful comments. 


\section{References}

Bertsekas, D. P. (1976). On the Goldstein-Levitin-Polyak gradient projection method. IEEE Transations on Automatic Control, 21, 174-184.

Bertsekas, D. P. (1999). Nonlinear programming (2nd ed.). Belmont, MA: Athena Scientific.

Brunet, J.-P. (2004). An NMF Program. Available online at http://www.broad.mit. $\mathrm{edu} / \mathrm{mpr} /$ publications/projects/NMF/nmf.m

Brunet, J.-P., Tamayo, P., Golub, T. R., \& Mesirov, J. P. (2004). Metagenes and molecular pattern discovery using matrix factorization. Proceedings of the National Academy of Science, 101(12), 4164-4169.

Calamai, P. H., \& Moré, J. J. (1987). Projected gradient methods for linearly constrained problems. Mathematical Programming, 39, 93-116.

Chu, M., Diele, F., Plemmons, R., \& Ragni, S. (2005). Optimality, computation and interpretation of nonnegative matrix factorizations. Preprint. Available online at http://www4.ncsu.edu/ mtchu/Research/Papers/nnmf.ps

Donoho, D., \& Stodden, V. (2004). When does non-negative matrix factorization give a correct decomposition into parts? In S. Thrün, L. Saul, \& B. Schölkopf (Eds.), Advances in neural information processing systems, 16. Cambridge, MA: MIT Press.

Gonzales, E. F., \& Zhang, Y. (2005). Accelerating the Lee-Seung algorithm for non-negative matrix factorization (Tech. Rep.). Houston, TX: Department of Computational and Applied Mathematics, Rice University.

Grippo, L., \& Sciandrone, M. (2000). On the convergence of the block nonlinear Gauss-Seidel method under convex constraints. Operations Research Letters, 26, 127-136.

Hoyer, P. O. (2002). Non-negative sparse coding. In Proceedings of IEEE Workshop on Neural Networks for Signal Processing (pp. 557-565). Piscataway, NJ: IEEE.

Hoyer, P. O. (2004). Non-negative matrix factorization with sparseness constraints. Journal of Machine Learning Research, 5, 1457-1469.

Lawson, C. L., \& Hanson, R. J. (1974). Solving least squares problems. Upper Saddle River, NJ: Prentice Hall.

Lee, D. D., \& Seung, S. (1999). Learning the parts of objects by nonnegative matrix factorization. Nature, 401, 788-791.

Lee, D. D., \& Seung, S. (2001). Algorithms for non-negative matrix factorization. In T. K. Leen, T. G. Dietterich, and V. Tresp (Eds.), Advances in neural information processing systems, 13 ( pp. 556-562). Cambridge, MA: MIT Press.

Lewis, D. D., Yang, Y., Rose, T. G., \& Li, F. (2004). RCV1: A new benchmark collection for text categorization research. Journal of Machine Learning Research, 5, 361397.

Lin, C.-J., \& Moré, J. J. (1999). Newton's method for large-scale bound constrained problems. SIAM Journal on Optimization, 9, 1100-1127.

McCormick, G. P., \& Tapia, R. A. (1972). The gradient projection method under mild differentiability conditions. SIAM Journal on Control, 10, 93-98.

Paatero, P. (1999). The multilinear engine-A table-driven, least squares program for solving multilinear problems, including the $n$-way parallel factor analysis model. J. of Computational and Graphical Statistics, 8(4), 854-888. 
Paatero, P., \& Tapper, U. (1994). Positive matrix factorization: A non-negative factor model with optimal utilization of error. Environmetrics, 5, 111-126.

Piper, J., Pauca, P., Plemmons, R., \& Giffin, M. (2004). Object characterization from spectral data using nonnegative factorization and information theory. In Proceedings of AMOS Technical Conference. Mavi, HI.

Powell, M. J. D. (1973). On search directions for minimization. Mathematical Programming, 4, 193-201.

Shepherd, S. (2004). Non-negative matrix factorization. Available online at http://www.simonshepherd.supanet.com/nnmf.htm

Xu, W., Liu, X., \& Gong, Y. (2003). Document clustering based on non-negative matrix factorization. In Proceedings of the 26th Annual International ACM SIGIR Conference (pp. 267-273). New York: ACM Press.

Received May 1, 2006; accepted September 27, 2006. 